\title{
Review: high dose and moderate dose oral anticoagulants reduce events in coronary artery disease but increase major bleeding and are no more effective than aspirin
}

Anand SS, Yusuf S. Oral anticoagulant therapy in patients with coronary artery disease: a meta-analysis.JAMA 1999

Dec 1;282:2058-67.

QUESTIONS: Do oral anticoagulants (OAs) reduce death, recurrent myocardial infarction (MI), and stroke and increase bleeding in patients with established coronary artery disease (CAD)? Do the effects vary with intensity of $O A$ and aspirin use?

\section{Data sources}

Studies published between 1960 and July 1999 were identified by searching Medline, EMBASE/Excerpta Medica, and Current Contents using combinations of terms related to OAs and vascular disease; reviewing bibliographies of relevant papers; and contacting experts and pharmaceutical companies.

\section{Study selection}

Randomised trials were selected if they included patients who had established CAD, used OAs, and continued treatment for $\geq 3$ months.

\section{Data extraction}

Data were extracted on baseline patient characteristics, intensity of OA treatment, time of initiation of treatment, duration of treatment, and number of patients discontinuing treatment.

\section{Main results}

30 reports of 31 trials were included in the analysis. Data were analysed by strata that were based on intensity of anticoagulation: high intensity OAs (international normalised ratio [INR] > 2.8, 20 trials); moderate intensity OAs (INR 2 to 3, 8 trials); and low intensity OAs (INR $<2,3$ trials). More patients who received high intensity OAs (16 trials) had reduced total mortality; fatal and non-fatal MI, stroke; and the combined end point of death, MI; or stroke than did control patients who did not receive aspirin. Patients receiving OAs had increased major bleeding (table). Moderate intensity OAs reduced fatal and non-fatal MI, and stroke but increased major bleeding more than did control treatment (4 trials) (table). High or moderate intensity OAs showed no more reduction in end points than did aspirin but increased major bleeding (7 trials) (table). Low intensity OAs plus aspirin had no more effect on any of the above mentioned outcomes than did aspirin alone (3 trials).

\section{Conclusions}

In patients with coronary artery disease, high intensity oral anticoagulants (OAs) reduce total mortality, myocardial infarction, and stroke but increase major bleeding; moderate intensity OAs reduce myocardial infarction and stroke but increase major bleeding. High or moderate intensity OAs increase bleeding but do not reduce end points more than aspirin. Low intensity OAs plus aspirin do not differ in effect from aspirin alone.

\section{COMMENTARY}

CAD is the leading cause of morbidity and mortality in the US. Patients with CAD are living longer and are a growing cohort that remains at risk for recurrent ischaemic events and death. ${ }^{1}$ The meta-analysis by Anand and Yusuf provides an important look at the use of OAs in CAD, but the evidence is not sufficient to recommend the routine use of OAs after MI given the justifiable and widespread use of aspirin. Only one third of the trials included in the analysis $(n=9)$ were published in the past decade; most were small trials done 20 to 40 years ago. Generalisations from these studies are problematic for 2 reasons. Firstly, the clinical management of $\mathrm{CAD}$ and the management of $\mathrm{OA}$ treatment has changed (ie, the widespread use of aspirin, the use of the INR for monitoring anticoagulant effect, the frequency of monitoring, the use of anticoagulant services, the use of portable prothrombin time monitors, and newer thrombolytics). Secondly, patients with CAD have changed. Differences in the comorbid conditions of these patients and the treatment of these conditions (eg, treatment of Helicobacter pylori in peptic ulcer disease) have simultaneously occurred. Thus, direct extrapolation to the present day is not practical.

Two important questions remain unanswered: which patients with CAD would benefit most from OAs in the presence of aspirin? What INR level is most beneficial? Currently, it is reasonable to conclude that long term use of OAs after MI can be recommended for secondary prevention of $\mathrm{MI}$ in patients unable to tolerate daily aspirin, patients with persistent atrial fibrillation, and patients with left ventricular thrombus. ${ }^{2}$ Preliminary results from the Combination Hemotherapy and Mortality Prevention (CHAMP) trial support this conclusion. ${ }^{3}$ Warfarin alone or in combination with aspirin at INR values $<2.0$ does not appear to be clinically effective in secondary prevention of MI.

Rebecca J Beyth, MD, MS Baylor College of Medicine Houston, Texas, USA

1 Hunink MG, Goldman L, Tosteson AN, et al. JAMA 1997;277:535-42.

2 Cairns JA, Theroux P, Lewis HD Jr, et al. Chest 1998;114:611S-33S.

3 Fiore LD, Ezekowitz MD. http://www.maveric.org/projects/study12.html.

Oral anticoagulants $(O A s) v$ control treatment in patients with established coronary artery disease ${ }^{*}$

\begin{tabular}{|c|c|c|c|c|c|}
\hline Outcomes & OA intensity and comparison & OAs & Control & Odds reduction $(95 \% \mathrm{CI})$ & NNT (CI) \\
\hline \multirow{2}{*}{ Death, MI, or stroke } & Moderate $v$ control & $31.3 \%$ & $33.7 \%$ & $16 \%(-34$ to 20$)$ & Not significant \\
\hline & & & & Odds increase (Cl) & NNH (Cl) \\
\hline \multirow[b]{2}{*}{ Major bleeding } & High $v$ control & $4.6 \%$ & $0.7 \%$ & $600 \%(440$ to 820$)$ & 30 (21 to 44$)$ \\
\hline & High or moderate $v$ aspirin & $3.7 \%$ & $1.0 \%$ & $1.4 \%(0.6$ to 2.6$)$ & $74(40$ to 170$)$ \\
\hline
\end{tabular}

*Abbreviations defined in glossary; NNTs and NNHs calculated from data in article. Follow up data not available.

$\dagger$ Approximate value calculated assuming a control event rate of $0.1 \%$.

Source of funding: no external funding.

For correspondence: Dr S S Anand, Hamilton General Hospital, McMaster Clinic, 237 Barton Street East, Hamilton, Ontario L8L 2X2, Canada.Fax +1905 5279642. 